\title{
El impacto de la crisis en las trayectorias laborales de las mujeres inmigrantes en España
}

\author{
Sara Moreno-Colom ${ }^{1}$; Pedro López-Roldán²
}

Recibido: 28 de julio de 2016 / Aceptado: 12 de diciembre de 2016

\begin{abstract}
El artículo presenta un análisis sobre el impacto de la crisis económica en la trayectoria laboral de las mujeres inmigrantes según su lugar de procedencia. El punto de partida teórico considera las influencias mutuas entre la tipología de modelos migratorios femeninos y la situación laboral de las mujeres inmigrantes en el contexto de crisis económica en España. Siguiendo la literatura especializada y en base a los datos de la Muestra Continua de Vidas Laborales relativos al período 2007-2013, se analizan y comparan las trayectorias laborales de las mujeres procedentes de Ecuador, Rumania y Marruecos. Se trata de tres casos paradigmáticos tanto por el volumen que representan entre la población inmigrante como porque permiten comparar distintas tradiciones migratorias, a saber, latinoamericana, africana y del este de Europa. Los resultados apuntan que, a diferencias de otras crisis, la actual tiene consecuencias distintas sobre la relación empleo y género que conviven con mecanismos individuales que simultáneamente reproducen y rompen los roles tradicionales de género.
\end{abstract}

Palabras clave: migraciones; género; empleo; crisis

\section{[en] The impact of the crisis on career paths of immigrant women in Spain}

\begin{abstract}
The aim of the paper is analyse the impact of the economic crisis in the career path of immigrant women according to their place of origin. The theoretical point of view considers the mutual influences between the typology of female migration patterns and the employment situation of immigrant women in the context of economic crisis in Spain. Based on data from the Muestra Continua de Vidas Laborales for the period 2007-2013, the paper compares the career paths of women from Ecuador, Romania and Morocco. They are three typical cases due the volume representing the immigrant population and they allow comparing different migration traditions (Latin American, African and Eastern Europe). The results suggest that the current economic crisis has consequences on the relation between employment and gender with some changes and continuities in traditional roles.
\end{abstract}

Keywords: migrations; gender; employment; crisis

Sumario: 1. Introducción. 2. Antecedentes y perspectiva. 3. Modelo de análisis y metodología. 4. Resultados. 4.1. La situación laboral. 4.2. La movilidad laboral. 5. Conclusiones. 6. Bibliografía

1 Centre d'Estudis Sociològics sobre la Vida Quotidiana i el Treball. Institut d'Estudis del Treball. Universitat Autònoma de Barcelona. Departamento de Sociología

sara.moreno@uab.cat

2 Centre d'Estudis Sociològics sobre la Vida Quotidiana i el Treball. Institut d'Estudis del Treball. Universitat Autònoma de Barcelona. Departamento de Sociología

Pedro.lopez.roldan@uab.cat 
Cómo citar: Moreno-Colom, S.; López-Roldán, P. (2018) "El impacto de la crisis en las trayectorias laborales de las mujeres inmigrantes en España”. Cuadernos de Relaciones Laborales, 36(1), 65-87.

\section{Introducción}

El artículo presenta un análisis sobre el impacto de la crisis económica en la trayectoria laboral de las mujeres inmigrantes según su lugar de procedencia. El fenómeno de la feminización de las migraciones ha dado lugar a numerosos estudios sobre la inserción y la situación laboral de las mujeres inmigrantes. En el caso español, el crecimiento de estos flujos migratorios se inicia a finales del siglo XX muy vinculado a la demanda de mano de obra femenina en determinados sectores, como el servicio doméstico y los cuidados. El estatus de mujer inmigrante aparece vinculado a determinadas ocupaciones desprestigiadas y estigmatizadas. Molpeceres (2012) caracteriza la situación laboral de las mujeres inmigrantes en España en términos de concentración sectorial y ocupacional, precariedad en las condiciones laborales y escasa movilidad. El conjunto de estas características se explica, en buena parte, por el modelo productivo y la estructura ocupacional española donde la tercerización, la segmentación y la segregación son aspectos relevantes (Recio y Banyuls, 2011). La destrucción de ocupación y la precarización que conlleva el contexto de crisis tiene una dimensión sectorial muy importante que introduce cambios en la situación laboral de los hombres y las mujeres inmigrantes. Los datos relativos a los primeros años de crisis mostraban tendencias contrarias a la pauta tradicional que afectó, sobre todo, a la población inmigrante: disminución del empleo masculino y precarización del empleo femenino rompiendo con la pauta del salario de la mujer como complemento en la familia (Miguélez et al., 2011; Moreno, 2014).

En este marco, el presente artículo fija su interés en el colectivo de mujeres inmigrantes asalariadas durante el período de crisis económica comprendido entre los años 2007-2013 con un doble objetivo: desarrollar un análisis longitudinal para captar el impacto de la crisis en las trayectorias laborales según el lugar de procedencia; y poner en relación las trayectorias laborales femeninas con las masculinas. Por un lado, la cuestión es relevante porque la perspectiva más frecuente en los análisis sobre la inmigración en el contexto de crisis se centra en los procesos de retorno y las situaciones de mayor vulnerabilidad (López de Lera y Pérez-Caramés, 2015). De manera complementaria, el artículo propone analizar los colectivos que han conseguido permanecer en el mercado laboral. En este sentido, se considerará la destrucción de ocupación, los mecanismos para permanecer en el mercado de trabajo y las condiciones laborales del empleo que resiste el impacto de la crisis. Por el otro lado, la mayoría de investigaciones centradas en la situación laboral ofrecen una foto fija sobre las condiciones laborales que tienen una limitada capacidad analítica. Contrariamente, se propone una aproximación longitudinal para el abordaje dinámico de la presencia en el mercado de trabajo a través de la construcción de diversos indicadores.

El punto de partida teórico considera las influencias mutuas entre la tipología de modelos migratorios femeninos y la situación laboral de las mujeres inmigrantes en el contexto de crisis económica en España. Siguiendo la literatura especializada y 
en base a los datos de la Muestra Continua de Vidas Laborales (MCVL) relativos al período 2007-2013, se analizan y comparan las trayectorias laborales de las mujeres procedentes de Ecuador, Rumania y Marruecos. Se trata de tres casos paradigmáticos tanto por el volumen que representan entre la población inmigrante en España como porque permiten comparar distintas tradiciones migratorias, a saber, latinoamericana, africana y del este de Europa.

El texto se estructura en tres apartados con el marco teórico, la descripción de la estrategia metodológica y la presentación de los resultados. Las conclusiones finales apuntan que, a diferencia de otras crisis, la actual tiene consecuencias distintas sobre la relación empleo, género y migraciones que, simultáneamente, reproducen y rompen con los roles tradicionales de género. Los modelos migratorios y los modelos de empleo condicionan las trayectorias laborales de las mujeres inmigrantes, al tiempo que los cambios observados con relación a su comportamiento en el mercado de trabajo sugerirían, más allá de los datos analizados, cambios parciales en las relaciones de género.

\section{Antecedentes y perspectiva}

El artículo toma como punto de partida los resultados obtenidos en dos estudios anteriores realizados con los datos de la $\operatorname{MCVL}(2007,2011)$ donde se comparan las trayectorias laborales de hombres y mujeres tomando en consideración la experiencia migratoria (Miguélez et al., 2011; Moreno, 2014; Miguélez y López-Roldán, 2014). Las conclusiones del primer estudio relativo al periodo anterior a la crisis dibujan tres tendencias en materia de empleo, género y migraciones. En primer lugar, se observa que las mujeres autóctonas tienen más fácil la promoción laboral que las mujeres inmigrantes. La segunda tendencia pone de manifiesto que las mujeres inmigrantes no son un todo homogéneo; factores como el lugar de procedencia, el nivel de estudios o el tiempo de permanencia en el mercado de trabajo modulan sus itinerarios laborales (Miguélez et al., 2011). En este sentido, destaca el caso de las mujeres peruanas con estudios superiores, pioneras en movilidad ascendente como también muestran los estudios de Escriva (2003) y Reher y Requena (2009). Finalmente, el análisis evidencia que la desigualdad de género entre la población inmigrante es menor que entre la población autóctona. Este mayor equilibrio entre hombres y mujeres inmigrantes tiene que ver con las diferencias dentro de los distintos colectivos de inmigrantes (lugar de procedencia, nivel estudios, edad) y con el hecho de compartir una peor situación de partida en el mercado laboral.

El segundo estudio analiza la incidencia del primer período de crisis, hasta el año 2011, en las trayectorias laborales de los hombres y las mujeres inmigrantes que en 2007 tenían un empleo (Miguélez y López-Roldán, 2014; Moreno, 2014). En este caso, se evidencia cómo las mujeres inmigrantes soportan mejor la crisis (en su primera etapa) que sus compatriotas masculinos: cada vez hay más mujeres ejerciendo el rol de principales mantenedoras económicas del núcleo familiar. Asimismo, se apunta cómo las mujeres inmigrantes sufren más la crisis en comparación con las mujeres autóctonas en términos de precariedad laboral, aunque menos en términos de pérdida del empleo. Como conclusión, se subraya la doble cara de la segregación horizontal: por un lado, un "mal menor" en tiempos de crisis que permite a las muje- 
res inmigrantes resistir como empleadas en los sectores feminizados del mercado de trabajo y mantener los ingresos según el modelo migratorio que ampara su situación. Por otro lado, se constata que los empleos que ocupan las mujeres duran menos tiempo y son más precarios, de modo que ellas están más sujetas a la inestabilidad laboral y tienen más difícil la cotización y el acceso a los sistemas de protección social (Moreno, 2014).

En estos estudios y en otros (Muñoz-Comet, 2016) se concluye que la crisis afecta especialmente a la inmigración con un aumento significativo del desempleo $\mathrm{y}$, en particular, del desempleo de larga duración, con trayectorias laborales donde no sólo se ha producido un bloqueo, sino que en muchos casos se ha significado un retroceso, aumentando las desigualdades y evidenciando que el origen geográfico es un factor determinante de las oportunidades laborales, de integración social y de asimilación. Distintos estudios señalan la dimensión sectorial de la crisis que se ha cebado con la ocupación de sectores tradicionalmente masculinizados como la construcción (Muñoz-Comet, 2012; OPAM, 2011). Paralelamente, se ha dado un crecimiento de sectores tradicionalmente feminizados como los servicios de atención y cuidados a la dependencia durante los primeros años de la crisis económica. Si bien los datos también recogen diferencias en la evolución de la ocupación de las mujeres inmigrantes y autóctonas, los análisis realizados hasta el momento apuntan que el impacto diferencial de la crisis económica sobre el empleo de los hombres y las mujeres, según el origen geográfico, tiene relación con la segregación horizontal (vinculada a la feminización de determinados sectores productivos) y la segregación vertical (relacionada con la dificultad femenina de promocionar laboralmente) del mercado de trabajo (OPAM, 2011). Y, como precisa Cebrián (2012), aunque se han reducido las diferencias en los niveles de participación laboral entre los hombres y las mujeres, todavía existen elementos diferenciadores. Básicamente, porque la inmigración femenina responde a una incorporación laboral más homogénea, en comparación a la masculina, con una mayor concentración y segregación ocupacional en sectores poco cualificados, desprestigiados y con un fuerte componente servil (Pajares, 2009; Vicente Torrado, 2014).

Otro aspecto evidenciado es el menor impacto de la destrucción de empleo sobre la población empleada bajo contratos indefinidos. Una situación que según Zugasti (2014) vive uno de cada dos trabajadores asalariados extranjeros siendo mayor la presencia de mujeres. Como explica esta misma autora, hasta el año 2008 los contratos indefinidos eran superiores en el colectivo de hombres inmigrantes, pero en 2010 aparece una feminización de la estabilidad del empleo. Las razones se encuentran, nuevamente, en la destrucción de puestos de trabajo en sectores masculinizados $\mathrm{y}$, también, en el impacto de cambios legislativos que ofrecen mayor protección a colectivos como el de las empleadas del hogar, fuertemente feminizado y con importante presencia extranjera. Según Zugasti (2014), el perfil de persona extranjera con contratado indefinido responde a una mujer, de 25 a 34 años, con bajo nivel educativo que se inserta en puestos con una baja incidencia de la primera fase de la crisis como el sector servicios.

En cualquier caso, el conjunto de estos estudios también pone de manifiesto importantes diferencias entre el colectivo de mujeres inmigrantes, especialmente según el lugar de procedencia. Molpeceres (2012) evidencia como la variable país de procedencia/nacionalidad resulta la más explicativa cuando se analiza la situación laboral de las mujeres inmigrantes. Otras investigaciones señalan la diversidad y la heterogeneidad de los procesos de movilidad laboral de las mujeres inmigrantes más 
allá de las dificultades estructurales del mercado de trabajo español fuertemente segmentado y segregado (Oso y Parella, 2012). Asimismo, también se muestra cómo las características demográficas de las personas migrantes tienen consecuencias tanto en las poblaciones de origen como en las sociedades de destino (Domingo, 2008a). Existe una variedad de modelos migratorios femeninos según el lugar de procedencia (Domingo, 2008b), de modo que las relaciones de género moldean los procesos migratorios e inciden en la toma de decisión de quién migra según las formas de inserción en el mercado de trabajo del país de acogida. En este sentido, Vicente y Setién (2005) defienden que resulta imprescindible la consideración del conjunto de factores sociales en origen y destino: aspiraciones individuales, condicionantes socioeconómicas y relaciones de género. Como explica Vicente Torrado (2014), los datos sobre la realidad migratoria desagregados por sexo se justifican porque las migraciones internacionales no pueden ser analizadas tomando únicamente como modelo la migración masculina. Hombres y mujeres inician el proyecto migratorio bajo diferentes oportunidades, tanto en los países de origen como de destino, que incluyen desde aspectos legales hasta motivaciones individuales o familiares pasando por la demanda del mercado de trabajo o los cambios demográficos del país de recepción. En definitiva, las relaciones de género conforman un rasgo constitutivo de los factores que explican la migración y ésta, simultáneamente, reconfigura las relaciones de género (Vicente Torrado, 2014).

La importancia de considerar las diferencias que existen dentro del colectivo de mujeres extranjeras en España en función de los proyectos migratorios, las trayectorias y las características individuales (nivel estudios, situación familiar, edad etc.) encuentra respaldo teórico con las propuestas de las desigualdades múltiples (Verloo, 1997 citado en Oso y Parella, 2012) y de la perspectiva interseccional (Crenshaw, 1989 citado en Oso y Parella, 2012). Ambas consideran que el género, la clase social, el lugar de origen o la misma condición jurídica de extranjera son variables que actúan de forma interrelacionada sobre las trayectorias laborales de las mujeres inmigrantes.

A partir de las aportaciones teóricas reseñadas en este apartado, el presente artículo plantea la necesidad de considerar las diferencias entre el colectivo de mujeres inmigrantes para analizar su trayectoria laboral durante el período de crisis. Como elemento explicativo central se introduce el lugar de procedencia dada la influencia atribuida a los distintos modelos migratorios para analizar la situación laboral en el país de acogida. En este sentido, siguiendo los planteamientos de otros estudios (Domingo, 2008a; Vienten Torrado, 2014), se toma el país de origen como indicador de los proyectos migratorios mayoritarios. Desde esta perspectiva, se defiende que la tipología de modelos migratorios femeninos es un factor clave para entender las diferencias en las trayectorias laborales de las mujeres inmigrantes en el contexto de crisis, al tiempo que este contexto puede introducir cambios en dicha tipología condicionada por las relaciones de género. Se parte de la idea que las relaciones de género moldean los procesos migratorios e inciden en la toma de decisión de quién migra según las formas de inserción en el mercado de trabajo del país de acogida. Si bien, en última instancia y como conclusión, se pretende argumentar que, en el contexto de crisis, los cambios en el mercado laboral también pueden introducir cambios en las relaciones de género según la distribución de los trabajos.

En los últimos años se ha evidenciado la progresiva feminización de algunas corrientes migratorias hasta llegar a alcanzar una proporción similar a la de los hombres 
(Domingo, 2008b; Vicente Torrado, 2014). Pero esta mayor presencia de mujeres en los flujos migratorios solo se da en algunas procedencias que, una vez identificadas, permiten dibujar distintos modelos migratorios. En este sentido, la investigación existente destaca diferencias relevantes entre las mujeres inmigrantes procedentes de Latinoamérica, países africanos y Europa del Este (Domingo, 2008b; Herrera et al., 2005; Molpeceres, 2012). Según estos planteamientos, Domingo (2008b) desglosa las procedencias y describe tres situaciones en las corrientes migratorias de los últimos años: predominio femenino con mayoría de mujeres que emigran; predominio masculino con la persistencia del viejo modelo migratorio y proporción equilibrada de ambos sexos.

Siguiendo la literatura especializada, este artículo compara la trayectoria laboral de las mujeres originarias de Ecuador, Marruecos y Rumania. Se consideran tres procedencias relevantes en cuanto a volumen y paradigmáticas en cuanto perfil migratorio siguiendo la tipología de Domingo (2008b). El caso de Ecuador coincide con circunstancias de predominio femenino en las corrientes migratorias. En la medida que predomina un tipo de migrante con responsabilidades familiares que se hace cargo del mantenimiento del hogar, la decisión de emigrar supone dejar la familia en el país de origen con toda la carga emocional que esto conlleva. En efecto, las mujeres originarias de Ecuador vienen a España, principalmente, por motivos económicos. A finales de los años 90 del siglo XX la emigración en Ecuador da un giro y se caracteriza por el protagonismo femenino y el escenario europeo (Herrera et al., 2005): las mujeres latinoamericanas satisfacen las necesidades del trabajo reproductivo en el mercado laboral. Dada la mayor facilidad por encontrar trabajo en el sector doméstico y de los cuidados, las mujeres ecuatorianas emigran a España como trabajadoras independientes o encabezando los proyectos migratorios de las familias. Posteriormente, la expansión económica del mercado español atraerá muchos hombres ecuatorianos en el sector de la construcción consiguiendo, en poco tiempo, mejores condiciones laborales que sus compatriotas. En cualquier caso, el perfil de mujer ecuatoriana se corresponde con un nivel educativo medio y un muy alto nivel de actividad laboral concentrado en las actividades del hogar, hostelería, comercio y servicios auxiliares.

Por el contrario, la migración de las mujeres magrebíes se inicia, mayoritariamente, en los procesos de reagrupación familiar. Es decir, la migración no constituye un proyecto propio sino vinculado al marido $\mathrm{y}$, por lo tanto, reproduce situaciones de supeditación económica porque el permiso de residencia de las mujeres no implica permiso de trabajo (Domingo, 2008a). Se trata de un caso con predominio masculino dada la influencia de la sociedad de origen que ejerce un fuerte control sobre las mujeres relegándolas a la reproducción y a la esfera privada. En la sociedad de acogida, el empleo femenino no siempre es una opción aceptada por los maridos. Así pues, el nivel de actividad laboral de las marroquíes suele ser bajo y su movilidad sectorial y ocupacional también, en parte, por el bajo nivel de estudios que tienen.

Finalmente, el caso de Rumania responde a la situación de proporciones más equilibradas entre hombres y mujeres que se da en la mayoría de las naciones europeas donde las diferencias de género existen pero son menos marcadas. Las mujeres originarias de Rumania vienen, al igual que las ecuatorianas, más por razones económicas que familiares. Los flujos migratorios de la población rumana son los más recientes y se han dado de forma intensiva en el tiempo, en pocos años llega mucha gente. Las características demográficas de las personas que han llegado se ajustan a 
las propias de cualquier flujo migratorio respecto a la juventud y con unas proporciones bastante equilibradas en cuanto al sexo, algo más elevadas para los hombres. Se trata de una inmigración que se ve influida por tres factores que parecerían afectar de igual forma a hombres y mujeres: la pertenencia a la Unión Europea que facilita los movimientos entre países y los traslados temporales; el contexto de crisis que restringe los empleos en la construcción y los servicios, sectores en los que se ubicaban numerosas personas rumanas; y la amplia y difusa presencia de personas rumanas en zonas rurales del interior (Domingo, 2008a). Según Molpeceres (2012), el perfil se corresponde a una mujer con estudios secundarios o superiores, con alto nivel de actividad y movilidad sectorial y ocupacional alta.

\section{Modelo de análisis y metodología}

Siguiendo la literatura especializada y utilizando como instrumento fundamental la Muestra Continua de Vidas Laborales (MCVL) ${ }^{3}$, se analizan y comparan las trayectorias laborales de las mujeres procedentes de Ecuador, Rumania y Marruecos con un doble objetivo: desarrollar un análisis longitudinal para captar el impacto de la crisis en las trayectorias laborales según el lugar de procedencia; y poner en relación las trayectorias laborales femeninas con las masculinas. La hipótesis de partida apunta la existencia de distintos cambios en las relaciones de empleo y género entre la población inmigrante.

Concretamente, el análisis realizado se fija en los datos de la MCVL del período 2007-2013. Desde el 2004, la MCVL ofrece datos relativos de la distribución de la población según diversas características laborales registradas administrativamente a través de la Seguridad Social, así como de su historia laboral desde que existen registros informatizados en un diseño de panel. A partir de estos distintos registros de afiliación al sistema de la Seguridad Social, la MCVL permite introducir elementos comparativos sobre las trayectorias laborales de los hombres y las mujeres según su procedencia. El análisis se realiza con los datos personales, de afiliación y de cotizaciones. Los microdatos de la MCVL se obtienen a partir de una muestra muy extensa, de alrededor de 1.200 .000 casos, diseñada tomando como población de referencia una definición amplia: todas las personas que han estado en situación de afiliado en alta, o recibiendo alguna pensión contributiva de la Seguridad Social en algún momento del año de referencia. Por tanto, cotizantes o pensionistas que, desde el punto de vista del ámbito temporal, incluye a todos los individuos que han estado en relación con la Seguridad Social en algún momento del año, no en una fecha fija, para facilitar así la presencia en la muestra de personas que trabajan regularmente pero que entran y salen de manera continuada de una situación de alta laboral.

Con el fin de responder a los objetivos planteados en el apartado anterior, se parte de la MCVL del año 2013, contexto de crisis económica y, a partir de éste, se mira retrospectivamente la situación respecto al 2007, contexto expansión económica. Se homogeneizan las poblaciones de ambas muestras permitiendo una comparación entre itinerarios laborales muy consistente, puesto que, al tratarse de datos de tipo

Sobre las características de los datos de la MCVL se puede consultar MTAS (2006), Durán (2007), García Pérez (2008), Lapuerta (2010), Moreno-Colom y López-Roldán (2011) o Miguélez et al. (2011). 
panel, es posible analizar las mismas personas en el periodo de crisis y en el periodo de expansión. Desde una perspectiva metodológica, el análisis longitudinal resulta pertinente para captar como la heterogeneidad y la diversidad dentro de los colectivos actúa en un contexto de cambio como representa el período de crisis económica. Dicho análisis permite superar la visión estática de los estudios que no contemplan la capacidad de agencia de las mujeres inmigrantes. Para acotar una fecha de referencia de los individuos se ha optado por considerar la última situación del año analizado centrándose específicamente en la población activa cotizante: ocupados (asalariados o autónomos) o parados. Finalmente, el Cuadro 1 presenta las principales variables del modelo de análisis trabajadas en los estudios que sirven de base para el presente artículo (López-Roldán, 2011; Miguélez y López-Roldán, 2014; Moreno, 2014) 4 .

Cuadro 1. Variables

\begin{tabular}{|c|c|c|}
\hline $\begin{array}{l}\text { Características estructurales } \\
\text { de los empleos }\end{array}$ & $\begin{array}{l}\text { Características de trayecto- } \\
\text { ria y movilidad }\end{array}$ & $\begin{array}{l}\text { Características individuales } \\
\text { de los trabajadores }\end{array}$ \\
\hline $\begin{array}{ll}\text { - Relación con la activi- } \\
\text { dad } \\
\text { - } \\
\text { - } \\
\text { Antigüedad } \\
\text { lación laboral } \\
\text { - } \text { Régimen de cotización } \\
\text { - Jornada laboral } \\
\text { - } \\
\text { - } \\
\text { - Tamaño de actividad } \\
\text { - } & \text { Territorio de la empresa } \\
\text { - } & \text { Base de cotización }\end{array}$ & $\begin{array}{ll}\text { - } & \text { Tiempo ocupado total no } \\
& \text { superpuesto } \\
\text { - } & \text { Tiempo ocupado total no } \\
& \text { superpuesto fijo } \\
\text { - } & \text { Tiempo ocupado total no } \\
& \text { superpuesto temporal } \\
- & \text { Tiempo total de desem- } \\
& \text { pleo no superpuesto con } \\
& \text { prestación y periodos sin } \\
& \text { relación } \\
- & \text { Paro de larga duración } \\
- & \text { Trayectorias de empleo } \\
- & \text { Movilidad ocupacional } \\
- & \text { Movilidad de ingresos } \\
- & \text { Movilidad sectorial } \\
- & \text { Movilidad territorial }\end{array}$ & $\begin{array}{ll}\text { - } & \text { Edad } \\
\text { - } & \text { Sexo } \\
\text { - } & \text { Origen geográfico } \\
\text { - } & \text { Nivel de estudios } \\
\text { - } & \text { Lugar de residencia }\end{array}$ \\
\hline
\end{tabular}

Elaboración propia.

Como toda fuente de información las ventajas que proporciona (muestra de gran tamaño, representativa de todo el año, datos longitudinales de la vida laboral completa, diseño de tipo panel) se contraponen a las desventajas (el hecho de ser registros administrativos, la complejidad de tratamiento de los datos, la falta de cobertura de algunos colectivos de la población activa e inactiva). Entre las limitaciones cabe resaltar, a efectos del análisis aquí realizado, la ausencia de trabajadores que no tienen sistema de previsión, los parados sin prestación y, sobre todo, el trabajo informal o sumergido donde se estima, en el año 2008, que un $10 \%$ de los inmigrantes residentes en España no tenía permiso de residencia (González-Enríquez, 2009). En lo fundamental, el retrato que emerge de los datos de la MCVL no debe verse alterado en el diagnóstico principal de los perfiles de empleo ni en las tendencias en el tiempo. No obstante, la mayor precariedad y vulnerabilidad de las situaciones del empleo

$4 \quad$ Una explicación más detallada y exhaustiva de la información utilizada se puede consultar en López-Roldán (2014: 28-66). 
no formal, al no estar presentes, morigeran el perfil de peor calidad del empleo del segmento secundario del mercado de trabajo. En el análisis tampoco podemos reflejar el fenómeno del abandono de la actividad laboral, de la emigración o el retorno migratorio, cuyo perfil no es aleatorio (Miyar-Busto y Muñoz-Comet, 2015) y nos centramos en las personas que se han mantenido en el periodo estudiado. No obstante, se trata de un colectivo de menor incidencia en los datos de la MCVL donde se observan altos niveles de estabilidad en la muestra (López-Roldán, 2014: 37 y 288).

Los resultados que se presentan a continuación analizan cómo ha transcurrido la trayectoria laboral de las mujeres originarias de Ecuador, Marruecos y Rumania que en el año 2007 formaban parte de la MCVL y en el año 2013 permanecen en la Seguridad Social como asalariadas con empleo, prestación o subsidio por desempleo. Concretamente, se trata de una población formada por 296.352 mujeres y 330.135 hombres. En ella, el colectivo de mujeres marroquíes representa el 0,8\% (2607), el colectivo ecuatoriano el 1,6\% (4873) y el colectivo rumano el 1,1\% (3354). La primera parte del análisis presenta una comparación descriptiva de las condiciones laborales que las mujeres tenían en 2007 y 2013 según el lugar de procedencia y en comparación a sus homólogos masculinos. El objetivo principal de esta parte es una primera aproximación a partir de la descripción del escenario anterior a la crisis y el escenario que resulta después de seis años de acumulación de la misma. Concretamente, se quieren analizar las condiciones laborales de las personas que en 2013 aparecían registradas en la Seguridad Social como asalariadas y que en 2007 formaban parte de la MCVL. Para ello, se consideran como variables dependientes: el tipo de relación laboral, el sector de actividad y el grupo de cotización. La segunda parte del análisis se focaliza en el estudio de la movilidad laboral. En este sentido, el análisis de los datos se fija en los movimientos en términos de escala de ingresos, seguridad contractual, movilidad ocupacional, movilidad sectorial y movilidad territorial. En este caso, se sigue con la lógica comparativa 2007-2013 y se consideran como variables dependientes: los días con contrato indefinido, los cambios de grupo de cotización, los cambios de sector y los cambios de territorio. Las técnicas de análisis utilizadas en la explotación de los datos son las propias del tratamiento estadístico descriptivo de relaciones bivariables.

\section{Resultados}

El periodo de crisis económica supone, desde sus inicios, menos empleo y más precariedad. Crece el paro y empeoran las condiciones laborales especialmente en el caso de los colectivos más vulnerables dentro del mercado de trabajo. A diferencia de otras crisis, las mujeres soportan mejor que los hombres los impactos negativos de la recesión económica, tanto en el caso de la población autóctona como de la inmigrante. Pero más allá de las grandes tendencias, los datos de la MCVL evidencian diferencias en la situación laboral de las mujeres inmigrantes según el lugar de procedencia que, a su vez, también introducen diferencias en comparación a sus homólogos masculinos. 


\subsection{La situación laboral}

La variable relación laboral permite analizar los episodios de afiliación a la Seguridad Social diferenciando las personas con empleo de las personas perceptoras de una prestación o subsidio de desempleo. Como era de esperar, los datos de la tabla 1 ponen de manifiesto que, durante el periodo 2007-2013, disminuyen las mujeres con empleo y crecen las mujeres que reciben algún tipo de prestación o subsidio, especialmente en el caso de las originarias de Marruecos. En este colectivo, destacan los porcentajes más bajos de relación laboral y más altos de subsidio de desempleo en comparación a la prestación. Es preciso matizar que las cifras de subsidios se pueden interpretar como un indicador de más tiempo acumulado sin empleo. El conjunto de estas diferencias se explicaría por la debilidad de su situación laboral durante el período de expansión puesto que el primer empleo que se destruye con la crisis es el más precario. En este sentido, se observa que la situación laboral de las mujeres marroquíes en 2007 ya acumulaba más paro (y más precariedad) en comparación a las ecuatorianas y rumanas. Tal y como muestran los datos relativos al 2013, los años de crisis amplían las diferencias en la situación laboral de las mujeres inmigrantes según su lugar de procedencia.

Tabla 1. Relación Laboral Mujeres Según Origen. España 2007-2013 (\%)

\begin{tabular}{|l|c|c|c|c|c|c|}
\hline \multirow{2}{*}{} & \multicolumn{2}{|c|}{ Ecuador } & \multicolumn{2}{c|}{ Marruecos } & \multicolumn{2}{c|}{ Rumania } \\
\cline { 2 - 7 } & 2007 & 2013 & 2007 & 2013 & 2007 & 2013 \\
\hline Relación laboral & 93,6 & 76,8 & 89,6 & 73,9 & 95,5 & 78,3 \\
\hline Prestación desempleo & 4,8 & 11,6 & 4,6 & 8,1 & 3,2 & 9,9 \\
\hline Subsidio desempleo & 1,5 & 11,6 & 5,8 & 18,0 & 1,3 & 11,8 \\
\hline
\end{tabular}

Total: 10.834 Fuente: Elaboración propia a partir de la MCVL (2007 y 2013)

Obviamente, en el caso de los hombres también disminuye la población ocupada y crecen los subsidios por desempleo pero, a diferencia de otras crisis, sucede con mayor intensidad que sus homólogas femeninas. Especialmente, destaca el caso de los ecuatorianos y los rumanos quienes tienen unos porcentajes de relación laboral más de diez puntos por debajo en comparación a las mujeres y de subsidio por desempleo más de siete puntos por encima. A la luz de estos datos, parece posible apuntar que, en ambos colectivos, ellas resisten mejor que ellos el impacto de la crisis en el mercado de trabajo. En cambio, en el caso de la población marroquí parece que el impacto de la crisis deja en una situación laboral similar a los hombres y a las mujeres. 
Tabla 2. Relación laboral hombres según origen. España 2007-2013 (\%)

\begin{tabular}{|l|c|c|c|c|c|c|}
\hline \multirow{2}{*}{} & \multicolumn{2}{|c|}{ Ecuador } & \multicolumn{2}{c|}{ Marruecos } & \multicolumn{2}{c|}{ Rumania } \\
\cline { 2 - 7 } & 2007 & 2013 & 2007 & 2013 & 2007 & 2013 \\
\hline Relación laboral & 91,3 & 63,9 & 89,1 & 70,3 & 96,3 & 68,3 \\
\hline Prestación desempleo & 8,4 & 16,9 & 9 & 9,4 & 3,4 & 13,7 \\
\hline Subsidio desempleo & 0,3 & 19,1 & 1,9 & 20,2 & 0,4 & 18,1 \\
\hline
\end{tabular}

Total: 16.874 Fuente: Elaboración propia a partir de la MCVL (2007 y 2013)

La dimensión sectorial de la crisis económica la destaca como un elemento clave para analizar las trayectorias laborales durante el período analizado ${ }^{5}$. La concentración durante los años de expansión económica de los hombres en la construcción y de las mujeres en los servicios explica la mayor pérdida de empleo masculino a partir del 2007. Asimismo, da cuenta del motivo por el que las mujeres sufren menos la destrucción de ocupación que acarrea el primer periodo de la crisis a pesar de la precariedad que caracteriza su situación laboral. Las mujeres inmigrantes se concentran en los sectores más precarios, inestables, temporales y desprestigiados como son las actividades del hogar y la hostelería. En este sentido, especialmente vulnerables son las mujeres originarias de América Latina y África.

A partir de los datos de la MCVL (Gráfico 1 a 6), se observa que las principales variaciones en la distribución de la población de referencia según sectores de actividad son: el incremento de las actividades del hogar frente a la disminución en los sectores de hostelería y el comercio donde tradicionalmente se ha ocupado una parte importante de las mujeres inmigrantes. Cabe recordar que a partir del 1 de enero de 2012 empezó el periodo para que las empleadas del hogar pasaran a formar parte del régimen general de la Seguridad Social (Real Decreto 1620/2011, 14 noviembre) lo que puede explicar, en parte, el incremento que experimenta este sector. Otro elemento explicativo se puede atribuir a la entrada en vigor de la Ley de Promoción de la Autonomía Personal y Atención a las personas con Dependencia 39/2006 donde se contempló distintas prestaciones económicas que, a la práctica, permitieron contratar a mujeres inmigrantes (Martínez, 2011) antes de los recortes presupuestarios. En cualquier caso, se observa un movimiento de mujeres de sectores donde tradicionalmente estaban ocupadas como hostelería y comercio hacia las actividades del hogar que tradicionalmente quedaban invisibilizadas en las estadísticas oficiales dado su carácter sumergido.

Más allá de las tendencias generales, el análisis pormenorizado de los subsectores que configuran el sector servicios apunta diferencias entre las mujeres según el país de procedencia. En este sentido, destaca el aumento de mujeres ecuatorianas en las actividades del hogar y en el sector sanitario reflejo de un modelo migratorio muy vinculado a la demanda del mercado de trabajo bajo la lógica de la mercantilización de los cuidados (Parella, 2003). Otro elemento diferencial se detecta en el sector

Las variaciones porcentuales durante el periodo analizado indican dónde se ha destruido empleo y dónde se ha reubicado parte de la población de referencia asalariada. En este sentido, los sectores que aparecen con un porcentaje mayor de asalariados en 2013 no son forzosamente sectores que han crecido, sino que pueden reflejar el efecto del decrecimiento de otros sectores. 
primario donde se observa una disminución porcentual del colectivo de rumanos, tradicionalmente con más presencia, frente al leve crecimiento de las mujeres ecuatorianas y marroquíes.

Gráfico 1. Sector actividad mujeres ecuatorianas. España 2007-2013 (\%)

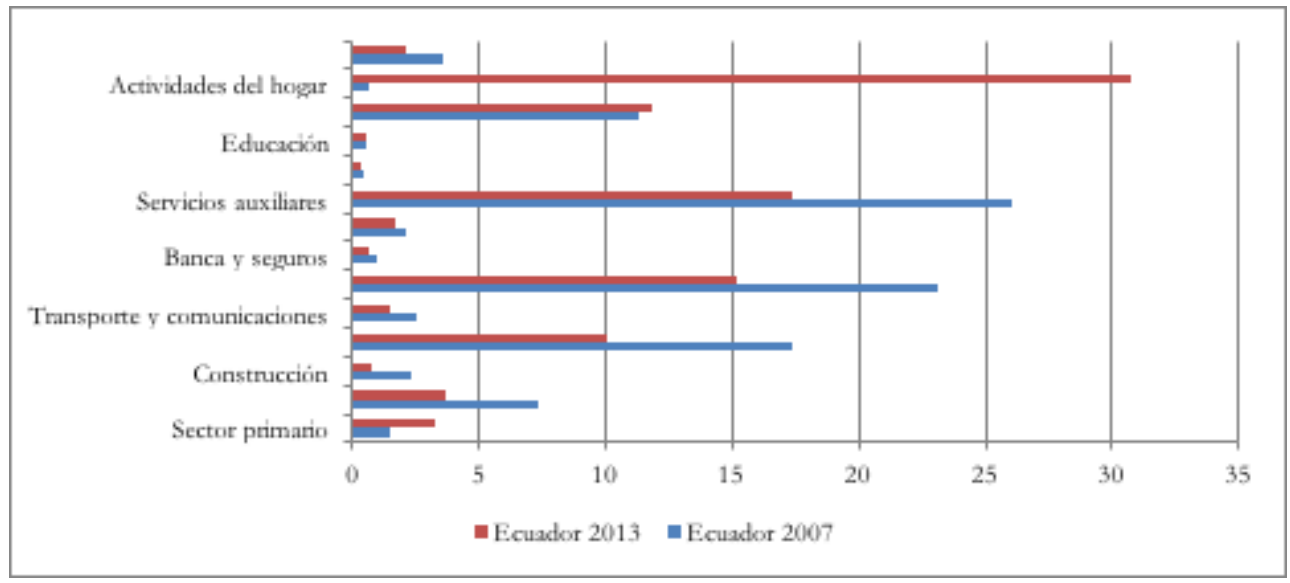

Fuente: Elaboración propia a partir de la MCVL (2007 y 2013)

Gráfico 2. Sector actividad mujeres marroquíes. España 2007-2013 (\%)

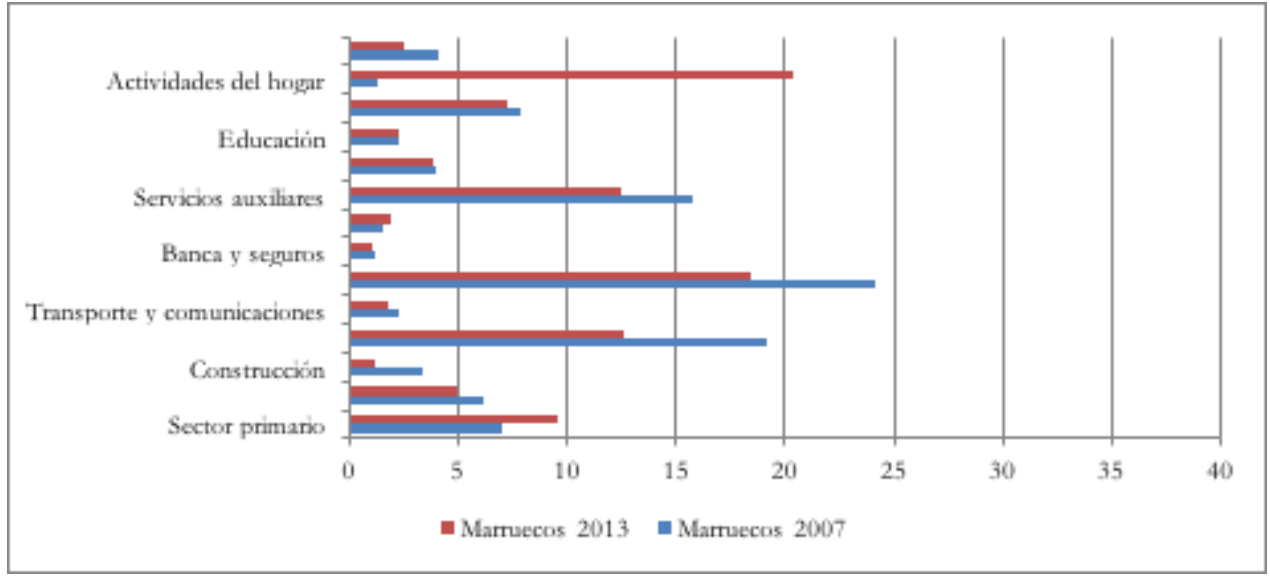

Fuente: Elaboración propia a partir de la MCVL (2007 y 2013) 
Gráfico 3. Sector actividad mujeres rumanas. España 2007-2013 (\%)

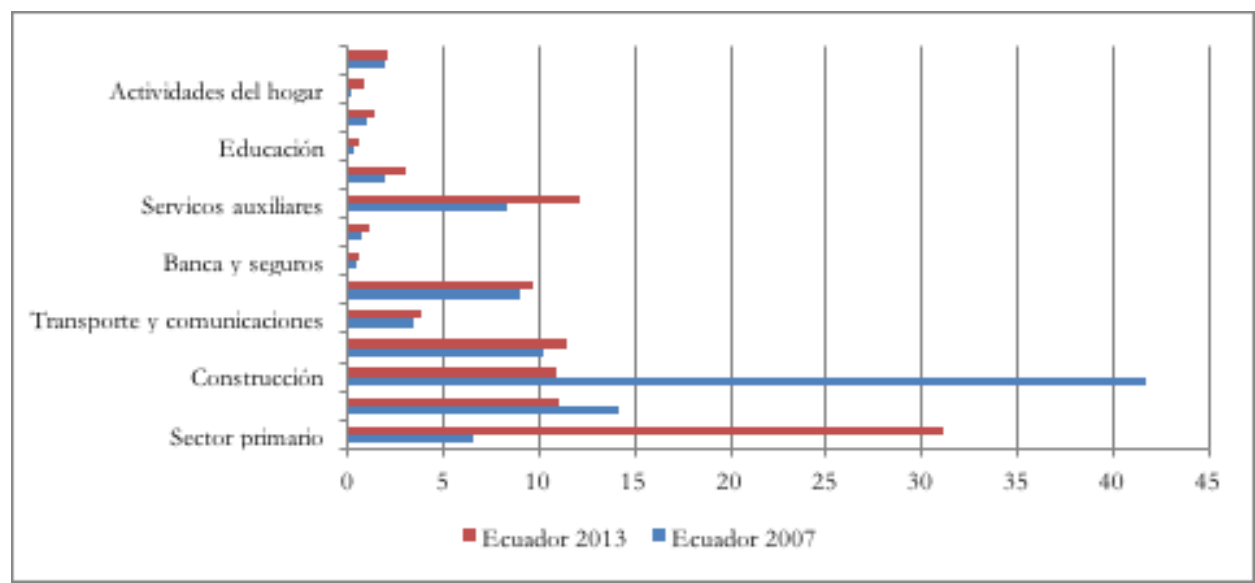

Fuente: Elaboración propia a partir de la MCVL (2007 y 2013)

La distribución sectorial de las mujeres inmigrantes contrasta con el caso de los hombres dada la destrucción de ocupación en el sector de la construcción, tradicional nicho laboral de la mano de obra inmigrantes masculina. En los tres colectivos analizados, disminuye más de 25 puntos porcentuales los ocupados en la construcción, mientras que el resto de sectores registra un leve crecimiento, en buena parte consecuencia del efecto composición que representa la movilidad intersectorial. A pesar de la coincidencia en las tendencias, se observa como elemento diferencial que en la industria disminuye levemente la presencia de ecuatorianos y marroquíes mientras que crece, también levemente, entre los rumanos.

Gráfico 4. Sector actividad hombres ecuatorianos. España 2007-2013 (\%)

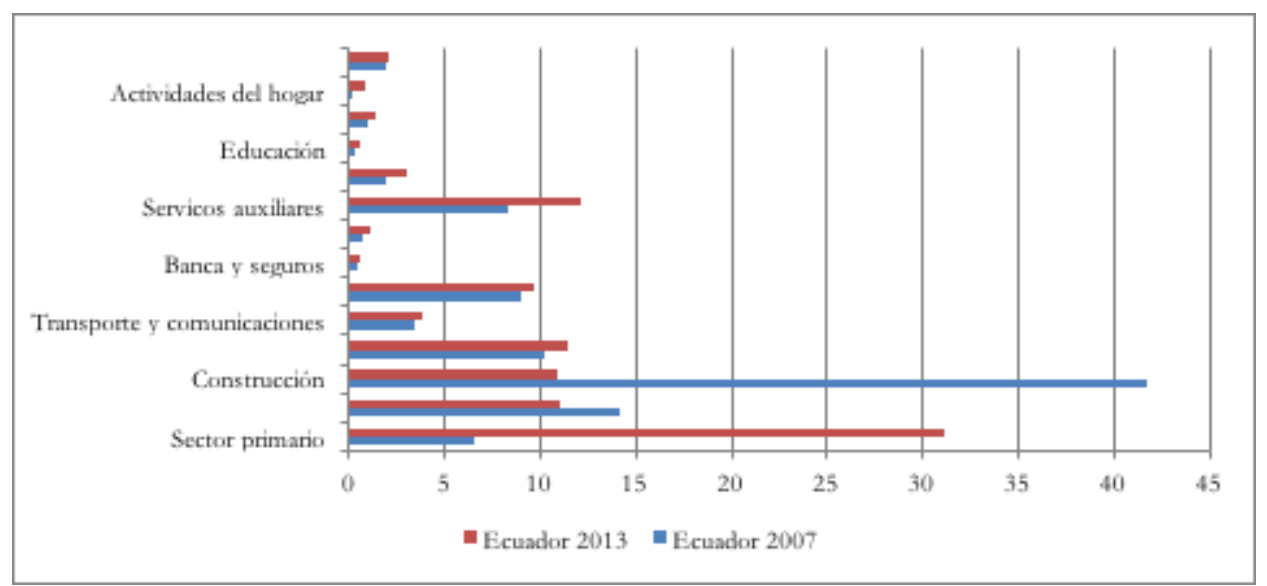

Fuente: Elaboración propia a partir de la MCVL (2007 y 2013) 
Gráfico 5. Sector de actividad hombres marroquíes. España 2007-2013 (\%)

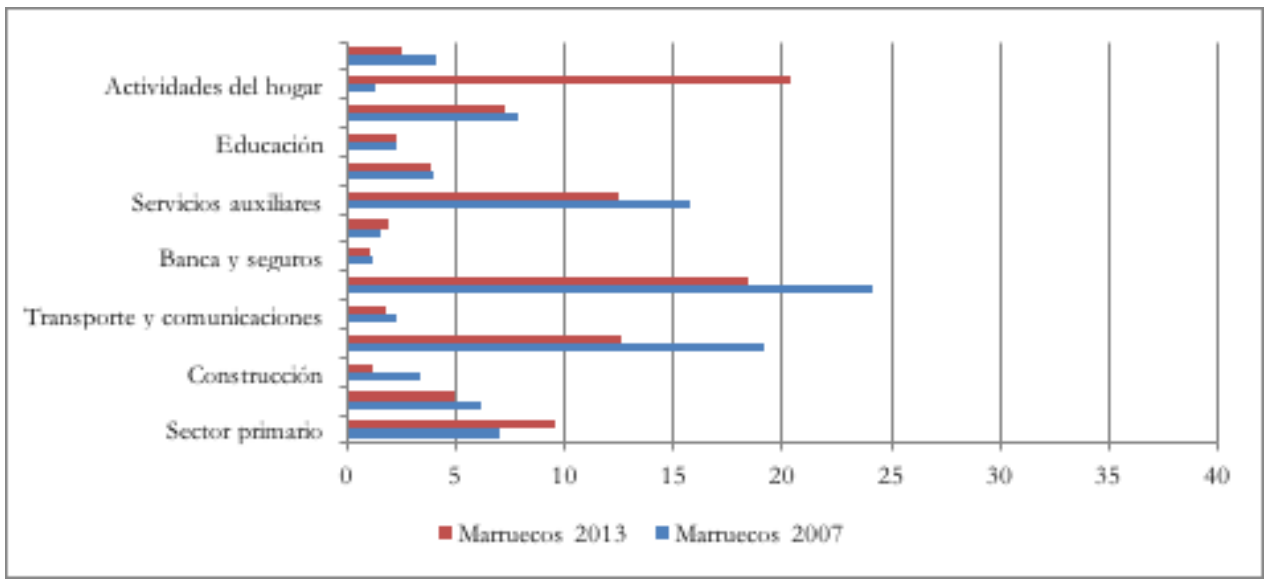

Fuente: Elaboración propia a partir de la MCVL (2007 y 2013)

Gráfico 6. Sector actividad hombres rumanos. España 2007-2013 (\%)

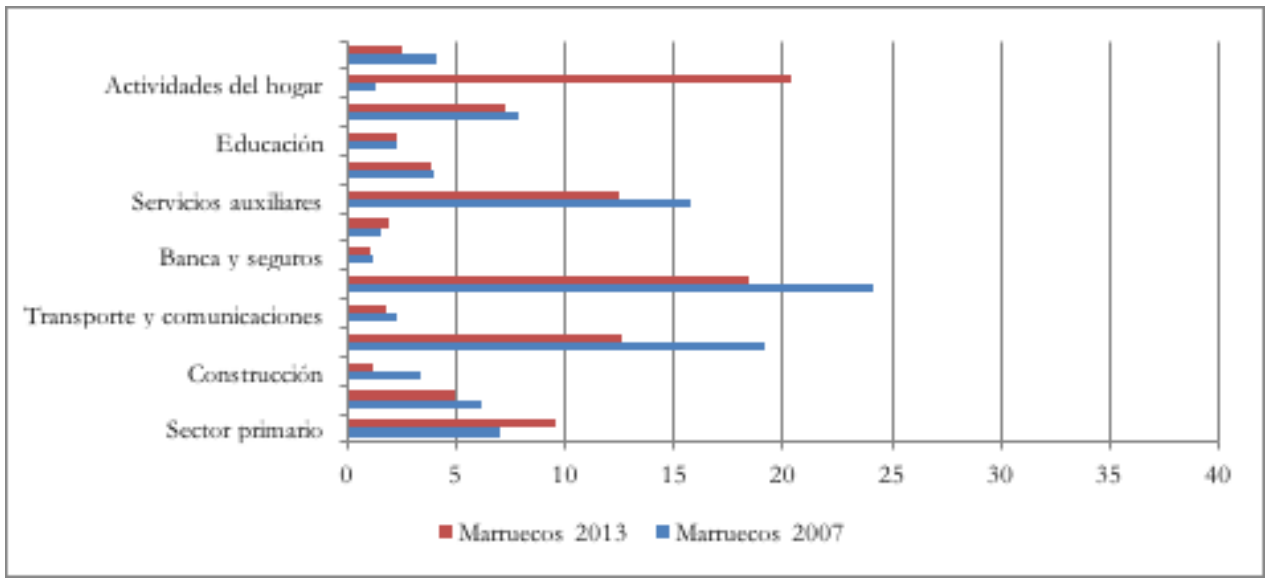

Fuente: Elaboración propia a partir de la MCVL (2007 y 2013)

Además de los cambios de sector, se analiza el impacto de la crisis en las condiciones laborales. Una primera aproximación se realiza a partir de la variable que clasifica la población en quintiles salariales de menor a mayor ingreso. Durante el período de crisis, se observa la tendencia a homogeneizar la distribución de la población femenina en quintiles salariales neutralizando, en buena parte, las diferencias según el lugar de procedencia. En la tabla 3 se observa que en los tres casos estudiados disminuye la presencia de mujeres en el primer y el tercer quintil, al tiempo que aumenta en el segundo y se mantiene bastante estable en el cuarto y el quinto. De entrada, los datos apuntarían una mejora de las mujeres en la escala salarial relativa a los tramos más bajos de salario y una pérdida en los tramos intermedios. Bajo esta 
óptica, las mujeres ecuatorianas aparecen como las mejor posicionadas. En el caso de las mujeres marroquíes cabe pensar que parte del grueso en el primer quintil se corresponde con las mujeres que reciben una prestación o subsidio por desempleo. Por el contrario, en el caso de las mujeres rumanas los porcentajes en los quintiles más bajos se deben relacionar con más precariedad en términos salariales. Aspectos que, en última instancia, guardan relación con el sector de actividad.

Tabla 3. Cotización por quintiles mujeres según origen. 2007-2013 (\%)

\begin{tabular}{|l|c|c|c|c|c|c|}
\hline & \multicolumn{2}{|c|}{ Ecuador } & \multicolumn{2}{c|}{ Marruecos } & \multicolumn{2}{c|}{ Rumania } \\
\hline & 2007 & 2013 & 2007 & 2013 & 2007 & 2013 \\
\hline Quintil 1 & 39,8 & 36,0 & 48,5 & 40,4 & 57,9 & 41,6 \\
\hline Quintil 2 & 19,7 & 38,0 & 15,1 & 31,5 & 14,2 & 33,0 \\
\hline Quintil 3 & 32,6 & 18,8 & 24,5 & 16,6 & 22,7 & 18,0 \\
\hline Quintil 4 & 7,3 & 6,6 & 7,3 & 7,0 & 4,6 & 6,5 \\
\hline Quintil 5 & 0,7 & 0,6 & 4,5 & 4,5 & 0,7 & 0,9 \\
\hline
\end{tabular}

Total: 10.834 Fuente: Elaboración propia a partir de la MCVL (2007 y 2013)

Aunque la escala salarial masculina refleja una distribución más equitativa de los hombres en los distintos quintiles (mayor presencia en los tramos medios y altos), la tendencia durante el período de crisis ha sido a la inversa que la femenina: crece la presencia en el primero y segundo quintil mientras que disminuye en el tercer, el cuarto y el quinto. En consecuencia, se pone de manifiesto una tendencia a reducir las diferencias entre hombres y mujeres, especialmente en el caso de las escalas salariales de los originarios de Ecuador y Marruecos.

Tabla 4. Cotización por quintiles hombres según origen. 2007-2013

\begin{tabular}{|l|c|c|c|c|c|c|}
\hline & \multicolumn{2}{|c|}{ Ecuador } & \multicolumn{2}{c|}{ Marruecos } & \multicolumn{2}{c|}{ Rumania } \\
\hline & 2007 & 2013 & 2007 & 2013 & 2007 & 2013 \\
\hline Quintil 1 & 12,6 & 32,5 & 17,5 & 40,5 & 32,9 & 30,5 \\
\hline Quintil 2 & 9,7 & 25,6 & 14,7 & 21,7 & 10,5 & 24,4 \\
\hline Quintil 3 & 47,4 & 25,2 & 44,0 & 21,1 & 35,6 & 28,2 \\
\hline Quintil 4 & 26,6 & 14,9 & 19,1 & 13,2 & 18,3 & 15,2 \\
\hline Quintil 5 & 3,7 & 1,9 & 4,6 & 3,5 & 2,7 & 1,7 \\
\hline
\end{tabular}

Total: 16.874 Fuente: Elaboración propia a partir de la MCVL (2007 y 2013)

\subsection{La movilidad laboral}

A partir de los datos descriptivos de la situación laboral de las mujeres inmigrantes, resulta de interés analizar el impacto de la crisis en su trayectoria laboral según el lugar de procedencia y en comparación con sus coetáneos masculinos. Este apartado presenta distintos indicadores de movilidad construidos a partir de la MCVL para el período 2007-2013. En primer lugar, se aborda la cuestión del nivel de seguridad 
contractual a partir de la proporción de días trabajados con contrato indefinido sobre el total de días trabajados a lo largo del tramo 2007-2013 (Moreno, 2014). La trayectoria laboral de las mujeres ecuatorianas acumula niveles de seguridad más elevados en comparación a las mujeres rumanas y marroquíes. Del mismo modo, la trayectoria laboral de las mujeres inmigrantes en su conjunto responde a unos niveles de seguridad más elevados que las de sus homólogos masculinos quienes registran más del 50\% de seguridad contractual baja, siendo menores las diferencias según el lugar de procedencia.

Tabla 5. Seguridad contractual según sexo y origen. España 2007-2013 (\% días indefinido)

\begin{tabular}{|l|c|c|c|c|c|c|}
\hline & \multicolumn{3}{|c}{ Mujeres } & \multicolumn{3}{c|}{ Hombres } \\
\hline & Ecuador & Marruecos & Rumania & Ecuador & Marruecos & Rumania \\
\hline Baja (menos 30\%) & 32,3 & 46,4 & 45,7 & 52,5 & 56,8 & 53,5 \\
\hline Media (30-80\%) & 32,8 & 24,3 & 30,6 & 25,8 & 21,8 & 25,8 \\
\hline Alta (más 80\%) & 34,9 & 29,2 & 23,7 & 21,7 & 21,3 & 20,7 \\
\hline
\end{tabular}

Total: 27.518 Fuente: Elaboración propia a partir de la MCVL (2007 y 2013)

El segundo indicador se construye con relación a la movilidad ocupacional según los cambios de grupo de cotización considerados como equivalentes a la categoría laboral. Con el fin de analizar hasta qué punto se promociona laboralmente durante el período señalado, se definen cinco categorías: desciende, no cambia, promoción baja, promoción media y promoción alta. De entrada, destaca la no movilidad de la mayoría de población que conserva su categoría laboral a lo largo de estos años. Se trataría de una tendencia lógica si se considera que las personas con más capacidad de resistir la destrucción de empleo son las más estables en el lugar de trabajo. En este sentido, se explicaría el caso de las mujeres marroquíes que, siendo las más vulnerables en el mercado de trabajo, las que logran conservar el empleo son aquellas que menos cambian de grupo de cotización. La segunda cuestión a destacar es que los porcentajes de movilidad ascendente superan ligeramente los de movilidad descendente en los tres grupos de mujeres, si bien las rumanas acumulan una mayor proporción de cambios seguidas de las ecuatorianas y las marroquíes. En cambio, en el caso de los hombres se reducen los porcentajes de no movilidad aun siendo la situación mayoritaria, especialmente entre los marroquíes al igual que en el caso de las mujeres. Pero esta mayor movilidad ocupacional masculina se traduce en peor promoción laboral si se compara con los porcentajes femeninos. En todos los grupos, los cambios descendentes y la promoción baja de los hombres son superiores a los de las mujeres. A pesar de ello, se observa que, en ambos casos, el colectivo originario de Ecuador acumula los mayores porcentajes de movilidad descendente. Una tendencia que parece guardar relación con la movilidad sectorial como se explica a continuación. 
Tabla 6. Cambio grupo cotización según sexo y origen. España 2007-2013 (\%)

\begin{tabular}{|l|c|c|c|c|c|c|}
\hline & \multicolumn{3}{|c|}{ Mujeres } & \multicolumn{3}{c|}{ Hombres } \\
\hline & Ecuador & Marruecos & Rumania & Ecuador & Marruecos & Rumania \\
\hline Desciende & 16 & 12,4 & 12,5 & 22,6 & 16,4 & 18,5 \\
\hline No cambia & 64,8 & 70,5 & 64,7 & 55,7 & 65,1 & 61,9 \\
\hline $\begin{array}{l}\text { Promoción baja } \\
(1-2)\end{array}$ & 11,2 & 10,4 & 13,6 & 15,8 & 11,6 & 15,1 \\
\hline $\begin{array}{l}\text { Promoción } \\
\text { media (3-5) }\end{array}$ & 7,5 & 6,2 & 8,3 & 5,6 & 6,3 & 4,2 \\
\hline $\begin{array}{l}\text { Promoción alta } \\
(6-9)\end{array}$ & 0,5 & 0,5 & 0,9 & 0,3 & 0,5 & 0,2 \\
\hline
\end{tabular}

Total: 27.518 Fuente: Elaboración propia a partir de la MCVL (2007 y 2013)

Miguélez y Godino (2014) argumentan que la movilidad sectorial puede venir motivada por la pérdida de empleo en otro sector o bien puede estar orientada a la búsqueda de unas mejores condiciones de trabajo. Mientras que la primera situación resulta típica en las personas que tienen empleo temporal o precario, la segunda situación mantiene relación con los intereses o los proyectos profesionales de quien cambia. De modo que los significados de la movilidad sectorial se pueden interpretar como una estrategia de supervivencia o como una estrategia de mejora.

En consonancia con la movilidad ocupacional, la trayectoria laboral de la mayoría de la población femenina no experimenta movilidad sectorial, si bien destacan, en segundo lugar, las trayectorias con movilidad sectorial de alta intensidad que registran tres o más cambios de sector. Más allá de las tendencias generales, se observa que las mujeres originarias de Ecuador experimentan más movilidad sectorial que el resto, siendo las mujeres marroquíes las que permanecen en mayor proporción en el mismo sector seguido de las rumanas. En este sentido, relacionando los cambios en los grupos de cotización con los cambios en los sectores, parece pertinente apuntar que la mayor movilidad sectorial de las mujeres ecuatorianas responde a estrategias de supervivencia en el mercado de trabajo. Aunque también es preciso apuntar que estas estrategias no son un sinónimo de una peor situación laboral en comparación a las mujeres marroquíes y rumanas. Como se ha visto, la situación laboral de las ecuatorianas implica mejores salarios y más seguridad. La condición de inmigrante económica que caracteriza el colectivo de mujeres ecuatorianas conlleva más predisposición al cambio y más disponibilidad a trabajar en cualquier sector. De este modo, la menor movilidad de las mujeres marroquíes no sería un indicador de mayor estabilidad sino reflejo de un proyecto migratorio distinto. Mientras que, en el caso de las mujeres rumanas, la menor movilidad sectorial (leve diferencia) podría estar relacionada con los sectores donde se ocupan, como el sector primario que se ha mostrado más resistente a la destrucción de ocupación. 
Tabla 7. Cambio de sector según sexo y origen. España 2007-2013 (\%)

\begin{tabular}{|l|c|c|c|c|c|c|}
\hline & \multicolumn{3}{|c|}{ Mujeres } & \multicolumn{3}{c|}{ Hombres } \\
\hline & Ecuador & Marruecos & Rumania & Ecuador & Marruecos & Rumania \\
\hline Sin cambio & 50,6 & 59,7 & 55,2 & 44,2 & 50 & 52 \\
\hline Un cambio & 13,2 & 12,7 & 13,1 & 15,6 & 13,5 & 14,5 \\
\hline Dos cambios & 12,5 & 9,9 & 11,4 & 12,3 & 10,7 & 12,5 \\
\hline $\begin{array}{l}\text { Tres o } \\
\text { más cambios }\end{array}$ & 23,8 & 17,6 & 20,3 & 27,9 & 25,8 & 21,5 \\
\hline
\end{tabular}

Total: 27.518 Fuente: Elaboración propia a partir de la MCVL (2007 y 2013)

Como era de esperar, las trayectorias laborales de los hombres también tienen más movilidad sectorial y de mayor intensidad en comparación a las trayectorias femeninas. Sin duda, se trata de una movilidad motivada por la pérdida de empleo en otro sector, en concreto, dada la destrucción de ocupación en la construcción. Pero de nuevo, se observa que el colectivo de ecuatorianos son los que más movilidad sectorial realizan y de mayor intensidad aunque, en 2007, no representaban los mayores porcentajes de ocupados en la construcción en comparación a los marroquíes y rumanas. Si se analizan estos datos con relación a los porcentajes de situación laboral (menos prestaciones y subsidios por desempleo), parece pertinente plantear que los ecuatorianos se mueven más que los marroquíes no como respuesta a la precariedad sino por tener más oportunidades. En cualquier caso, se observa que las diferencias de género con relación a la movilidad sectorial se reducen respecto a las diferencias relativas a la movilidad ocupacional. Ello se puede explicar por el hecho de que, en el contexto de crisis, muchas mujeres asumen el rol de principal proveedora del hogar como respuesta al paro masculino.

Finalmente, el cuarto indicador aborda la cuestión de la movilidad territorial. En este sentido, Miguélez y Godino (2014) constatan que dicha movilidad territorial es superior a nivel provincial que regional antes y durante la crisis, predominando la movilidad de baja intensidad (un solo cambio). La cuestión interesante es saber quién se mueve en tiempos de crisis. Los datos ponen de manifiesto que la población de referencia, mayoritariamente, no se mueve. En parte, resulta comprensible al recordar que se trata de personas que en 2007 formaban parte de la MCVL y que en 2013 permanecen como asalariadas (con empleo, prestación o subsidio). A grandes rasgos, se confirma que la movilidad territorial es masculina y provincial. Más concretamente, se observa que las mujeres rumanas se mueven más que las ecuatorianas y marroquíes, mientras que los hombres marroquíes se mueven más que los ecuatorianos y rumanos. En ambos casos, se puede relacionar la movilidad territorial con alta intensidad: entre los que se mueven más predomina la categoría tres o más cambios. La importancia de la movilidad territorial de las mujeres rumanas se podría explicar por su condición de inmigrante económica que comparten con las ecuatorianas, pero, sobre todo, por su situación familiar. Distintos estudios subrayan la juventud y ausencia de responsabilidades familiares como elementos característicos de uno de los perfiles de los inmigrantes rumanos. Dichas condiciones son aspectos que facilitan la movilidad territorial, especialmente en el caso de las mujeres. Por el contrario, el caso de los hombres marroquíes, aparte de responder a su condición de 
inmigrante económico, es preciso interpretarla como una estrategia de respuesta al paro y la precariedad de un proyecto migratorio fuertemente marcado por el imaginario "male breadwinner".

Tabla 8. Cambio cc.Aa según sexo y origen. España 2007-2013 (\%)

\begin{tabular}{|l|c|c|c|c|c|c|}
\hline & \multicolumn{3}{|c|}{ Mujeres } & \multicolumn{3}{c|}{ Hombres } \\
\hline & Ecuador & Marruecos & Rumania & Ecuador & Marruecos & Rumania \\
\hline Sin cambio & 88,9 & 89,8 & 81,9 & 72,8 & 71,1 & 74,9 \\
\hline Un cambio & 4,8 & 4,8 & 6,8 & 9,1 & 9,4 & 9,5 \\
\hline Dos cambios & 3,4 & 3,2 & 4,5 & 8,2 & 7,3 & 7,4 \\
\hline $\begin{array}{l}\text { Tres o } \\
\text { más cambios }\end{array}$ & 3 & 2,2 & 6,7 & 9,9 & 12,2 & 8,2 \\
\hline
\end{tabular}

Total: 27.518 Fuente: Elaboración propia a partir de la MCVL (2007 y 2013)

Tabla 9. Cambio provincias según sexo y origen. España 2007-2013

\begin{tabular}{|l|c|c|c|c|c|c|}
\hline & \multicolumn{3}{|c|}{ Mujeres } & \multicolumn{3}{c|}{ Hombres } \\
\cline { 2 - 7 } & Ecuador & Marruecos & Rumania & Ecuador & Marruecos & Rumania \\
\hline Sin cambio & 87,3 & 84,5 & 77,7 & 69,5 & 64,8 & 68 \\
\hline Un cambio & 5,4 & 6,6 & 7,9 & 9,3 & 10,6 & 10,4 \\
\hline Dos cambios & 3,8 & 4,8 & 5,9 & 9,1 & 8,9 & 9,3 \\
\hline $\begin{array}{l}\text { Tres o más } \\
\text { cambios }\end{array}$ & 3,5 & 3,2 & 8,5 & 12,1 & 15,7 & 12,2 \\
\hline
\end{tabular}

Total: 27.518 Fuente: Elaboración propia a partir de la MCVL (2007 y 2013)

\section{Conclusiones}

La creciente feminización de las migraciones ha facilitado la introducción de nuevos instrumentos analíticos para conocer las causas que originan los proyectos migratorios de las mujeres y su incidencia en las relaciones de género. Uno de los factores explicativos recae en las demandas del mercado de trabajo de los países de acogida. Por esta razón, resulta interesante analizar el impacto de la crisis económica en la trayectoria laboral de las mujeres inmigrantes en España. Desde esta perspectiva, el artículo se fija en el colectivo de asalariadas durante el período acumulado de crisis económica (2007-2013) con un doble objetivo: desarrollar un análisis longitudinal para captar el impacto de la crisis en las trayectorias laborales según el lugar de procedencia y poner en relación las trayectorias laborales femeninas con las masculinas. Las diferencias encontradas van en la línea de la hipótesis planteada acerca de la influencia de los modelos migratorios femeninos sobre las trayectorias laborales de las mujeres inmigrantes en el contexto de crisis.

Los datos presentados evidencian que el impacto de la crisis económica en la trayectoria laboral de las mujeres inmigrantes mantiene diferencias según su lugar de 
procedencia. Por un lado, la trayectoria laboral de la mujer inmigrante viene marcada por la vulnerabilidad de un grupo cuya situación laboral se caracteriza en términos de concentración sectorial y ocupacional, precariedad en las condiciones laborales y escasa movilidad (Molpeceres, 2012). Por otro lado, se apunta que dichas trayectorias laborales también están condicionadas por la tipología de las migraciones femeninas. En este sentido, la condición de inmigrante económica de las mujeres originarias de Ecuador y Rumania frente a la reagrupación familiar que motiva la llegada de la mayoría de mujeres marroquíes explica algunas diferencias en la situación y movilidad laboral. La trayectoria laboral de las mujeres ecuatorianas durante el período acumulado de crisis se caracteriza por mantener cierta estabilidad salarial, seguridad contractual media-alta, movilidad ocupacional descendente o de baja promoción, intensa movilidad sectorial y escasa movilidad territorial. La trayectoria laboral de las mujeres rumanas se caracteriza por mantener cierta estabilidad salarial, seguridad contractual baja-media, menor movilidad ocupacional descendente o de baja promoción, menor movilidad sectorial y mayor movilidad territorial. La trayectoria laboral de las mujeres marroquíes, se caracteriza por mantener cierta estabilidad salarial, seguridad contractual baja-media y poca movilidad ocupacional, sectorial y territorial.

El peso del lugar de procedencia apunta la importancia que ejercen los modelos migratorios femeninos en la situación laboral de las mujeres. Las mujeres ecuatorianas fueron las primeras en llegar a España encabezando proyectos migratorios económicos. El tiempo de permanencia en el mercado laboral junto la disponibilidad para el empleo son aspectos a considerar para explicar su mayor arraigo en el territorio y mayor movilidad sectorial. Hay indicios (seguridad contractual, quintiles salariales) que invitan a pensar que el cambio de sector responde a una estrategia de supervivencia fruto de una mayor capacidad de cambio en comparación a las mujeres marroquíes y rumanas. En este sentido, el menor tiempo de permanencia de las mujeres rumanas (llegan más tarde a España encabezando y compartiendo proyectos migratorios económicos con sus parejas) supone menor capacidad de resistir en un mercado laboral delimitado. Para ellas, la movilidad territorial parece una estrategia más eficaz en una situación laboral más precaria (con menor seguridad contractual y más bajos salarios). Finalmente, la vulnerabilidad laboral de las mujeres marroquíes se puede relacionar con un proyecto migratorio vinculado al reagrupamiento familiar, lo que conlleva menor disponibilidad para el empleo y menor predisposición para el cambio dentro del mercado de trabajo.

En conclusión, parece posible apuntar que las mujeres inmigrantes tienen distintas capacidades de respuesta ante el impacto que la crisis ejerce en su situación laboral. Tanto el impacto como las capacidades de respuesta se dan, en buena parte, en función del proyecto migratorio. El impacto es mayor en el caso de las marroquíes con una situación más vulnerable en el mercado de trabajo (más paro, menos salario, más temporalidad, menos movilidad ocupacional) y menor en el caso de las mujeres ecuatorianas con más tiempo de permanencia y mejor situación laboral (menos paro, menos temporalidad más movilidad ocupacional). La situación intermedia la protagonizan las mujeres rumanas. Como causa y consecuencia, se apuntan la diferente capacidad de respuesta según la trayectoria migratoria y el ciclo vital. Mientras que las mujeres ecuatorianas resisten en el mercado de trabajo gracias a la movilidad sectorial, entre las mujeres rumanas destaca la estrategia de la movilidad territorial. Al margen quedan las marroquíes con escasa capacidad de respuesta. Así pues, se 
observa que la movilidad en las trayectorias laborales de las mujeres inmigrantes se da en sentido inverso a su vulnerabilidad laboral.

Finalmente, los datos presentados invitan a plantear hasta qué punto los cambios en el mercado de trabajo influyen las relaciones de género siguiendo los planteamientos de algunos autores (Domingo, 2008a; Vicente Torrado, 2014). Asumiendo los límites de la contrastación empírica realizada para inferir directamente transformaciones en los roles de género, se sugiere que los cambios relativos a los comportamientos en el mercado de trabajo permiten esbozar, a modo de prospectiva, cambios parciales en las relaciones de género. La dimensión sectorial de la crisis económica durante su primer período explica por qué las trayectorias laborales de los hombres acumulan más paro, mayor pérdida salarial, más temporalidad, más movilidad ocupacional descendente, más movilidad sectorial y más movilidad territorial. El impacto negativo de la crisis es mayor en la situación laboral de los hombres de manera que en todos los colectivos analizados se reducen las diferencias de género a lo largo de los años 2007-2013. En este sentido, destaca la mejor resistencia de las mujeres ecuatorianas y rumanas, así como la casi "igualdad" entre el colectivo marroquí. La capacidad de respuesta en el caso de los hombres sigue patrones parecidos entre los ecuatorianos y rumanos, pero distinto entre los marroquíes con mayor movilidad territorial. En consonancia con el menor peso de la condición de inmigrante económica de las mujeres siguiendo el modelo migratorio de predominio masculino, los hombres asumen su papel de "male breadwinner". En cambio, la mayor capacidad de resistencia de las ecuatorianas y rumanas en el contexto de crisis las sitúa en el rol de principal proveedora del hogar como respuesta al paro masculino, en consonancia con los modelos de predominio femenino y proporción equilibrada respectivamente.

El conjunto de estas tendencias confirma que, a diferencias de otras crisis, la actual tiene consecuencias distintas sobre la relación empleo y género que conviven con mecanismos individuales que simultáneamente reproducen y rompen los roles tradicionales de género. Como explica Vicente Torrado (2014), las relaciones de género conforman un rasgo constitutivo de los factores que explican la migración y ésta, simultáneamente, reconfigura las relaciones de género. Si bien es cierto que la literatura especializada recuerda que históricamente la salida de las crisis económicas ha supuesto más trabajo invisible de las mujeres y más temprana recuperación del empleo masculino, en el caso de la población inmigrante los datos analizados abren algunos interrogantes. En este sentido, se sugiere que el contexto de crisis introduce cambios en los tres modelos de corrientes migratorias definidos por Domingo (2008a) cuando se observa la situación laboral de las mujeres en comparación a los hombres. Dichos cambios se podrían resumir según el papel de la mujer: ecuatorianas "de nuevo, jefas del hogar"; marroquíes "nuevas jefas de hogar"; y rumanas "trayectorias igualitarias". En cualquier caso, de confirmarse y consolidarse estos roles, es preciso señalar la paradoja que supone observar como las trayectorias laborales descritas permiten revisar las relaciones de género en la familia al tiempo que la situación de las mujeres en el mercado laboral refuerza los estereotipos sexistas. Con todo, se confirman las contradicciones de la doble cara de la segregación horizontal cuando se analizan las trayectorias laborales de las mujeres inmigrantes. 


\section{Bibliografía}

Cebrián, I. (2012). "La situación de las mujeres en el mercado de trabajo antes y durante la crisis según la Muestra Continua de Vidas Laborales". Cuadernos del Mercado de Trabajo. 8: 53-60.

Domingo, C. (2008a). “Características demográficas de los migrantes búlgaros y rumanos en España”. Cuadernos de Geografía. 84: 195-212.

Domingo, C. (2008b). "Inmigración femenina en España y relaciones de género". SEMATA, Ciencias Sociales y de Humanidades. 20: 71-92.

Domínguez Mujica, J. (2015). "La decisión de retornar en tiempos de crisis. Una perspectiva comparada en inmigrantes ecuatorianos y rumanos en España". Migraciones. 37: 171194.

Durán, A. (2007). "La Muestra Continua de Vidas Laborales de la Seguridad Social”. Madrid: Revista del Ministerio de Trabajo y Asuntos Sociales, Extra Seguridad Social.

Escriva, A. (2003). "Inmigrantes peruanas en España. Conquistando el espacio laboral extra doméstico". Revista Internacional de Sociología. 36: 59-83.

García Pérez, J. I. (2008). La muestra continua de vidas laborales: una guía de uso para el análisis de transiciones. Revista de Economía Aplicada, XVI, E-1, 5-28. (En línea). http:// www.revecap.com/revista/numeros/e1/pdf/garcia.pdf

González-Enríquez, C. (2009). Undocumented Migration. Counting the Uncontable. Data and Trends across Europe. Country Report Spain. European Commission.

Herrera, G.; Carrillo, M.C. y Torres, A. (2005). La migración ecuatoriana, transnacionalismo, redes e identidades. Ecuador: Flacso.

Lapuerta, I. (2010). Claves para el trabajo con la Muestra Continua de Vidas Laborales. DemoSoc Working Paper, 2010-37. Departament de Ciències Polítiques i Socials, Universitat Pompeu Fabra. (En línea). http://www.recercat.net/bitstream/2072/86913/1/DEMOSOC37.pdf.

Martínez, R. (2011). "La reorganización de los cuidados familiares en un contexto de migración internacional”. Cuadernos de Relaciones Laborales, 29 (1): 93-123.

Miguélez, F. y Godino, A. (2014). “¿Movilidad territorial y sectorial como respuesta a la crisis?” En Miguélez, F. y López-Roldán, P. (Coord.). Crisis, empleo e inmigración en España. Un análisis de las trayectorias laborales. (145-177) Bellaterra: UAB.

Miguélez, F. y López-Roldán, P. (Coord.) (2014). Crisis, empleo e inmigración en España. Un análisis de las trayectorias laborales. Bellaterra (Barcelona): UAB.

Miyar-Busto, M., y Muñoz-Comet, J. (2015). "La salida de inmigrantes en contextos de declive económico ¿Cambia la autoselección durante fases recesivas?”. Revista Española de Investigaciones Sociológicas, 152 (1), 87-102.

MTAS (2006). La muestra continua de vidas laborales. Madrid: Ministerio de Trabajo y Asuntos Sociales. Colección Informes y Estudios, Serie Seguridad Social, 24.

Molpeceres, L. (2012). "Situación laboral de las mujeres inmigrantes en España”. Cuadernos de Relaciones Laborales, 30 (1): 91-113.

Moreno-Colom S y López-Roldán P (2011). "La Muestra Continua de Vidas Laborales: posibilidades y limitaciones. Aplicación al estudio de la ocupación de la población inmigrante". Metodología de Encuestas, 13: 7-32.

Moreno-Colom S y López-Roldán P (2014). “Trayectorias laborales, género e inmigración: la doble cara de la segregación horizontal". En AUTORES (Coord.). Crisis, empleo e inmigración en España. Un análisis de las trayectorias laborales. (145-177) Bellaterra: UAB. 
Moreno-Colom S y López-Roldán P (2011): Trayectorias laborales de los inmigrantes en España. Barcelona: Obra Social de la Caixa.

Muñoz Comet, J. (2012). "Evolución del empleo y el paro de las mujeres inmigrantes en el mercado de trabajo español. Impacto de la actual crisis económica". Cuadernos de Relaciones Laborales, 30 (1): 115-137.

Muñoz Comet, J. (2016). Inmigración y empleo en España. De la expansión a la crisis económica. Madrid: Centro de Investigaciones Sociológicas.

Pajares, M. (2009). Inmigración y mercado de trabajo. Informe 2009. Madrid: OPI.

Parella, S. (2003). Mujeres, inmigrantes y trabajadoras. La triple discriminación. Barcelona: Anthropos.

OPAM (2011). Las mujeres inmigrantes en el mercado laboral español. Tema OPAM, 5.

Oso, L. y Parella, S. (2012). "Inmigración, género y mercado de trabajo: una panorámica de la investigación sobre la inserción laboral de las mujeres inmigrantes en España". Cuadernos de Relaciones Laborales, 30 (1): 11-44.

Recio, A. y Banyuls, J. (2011). "Crisis y modelos nacionales de empleo: la experiencia de diez países europeos en la crisis”. Revista de Economía Crítica, 11: 173-184.

Reher, D. y Requena, M. (eds.) (2009). Las múltiples caras de la inmigración en España. Madrid: Alianza Editorial.

Vicente, T. y Setién, M. L. (2005). "Modelos migratorios femeninos”. En Julia González y María Luisa Setién (eds.). Diversidad migratoria. Distintos protagonistas, diferentes contextos, Universidad de Deusto: Bilbao.

Vicente Torrado, T. L. (2014). "El panorama migratorio internacional: una mirada desde el género". Sociedad y Equidad: Revista de Humanidades, Ciencias Sociales, Artes y Comunicaciones, 6.

Zugasto, N. (2014). "Incidencia de la crisis en el empleo extranjero. Evidencias a partir de las transiciones laborales de trabajadores indefinidos". Papers, 99(2): 285-306. 